\title{
Mavridis' Area Targeting for Deep Brain Stimulation in Psychiatric Disorders: Stereotactic Microanatomy and Technical Aspects
}

\author{
Ioannis N. Mavridis, ${ }^{1, *}$ \\ ${ }^{1}$ Department of Neurosurgery, 'K.A.T.-N.R.C.' General Hospital of Attica, Athens, Greece \\ "Corresponding author: Ioannis N. Mavridis, Department of Neurosurgery, 'K.A.T.-N.R.C.' General Hospital of Attica, Kifissia, Athens, Greece. E-mail: pap-van@otenet.gr
}

Received 2015 October 28; Revised 2016 May 07; Accepted 2016 May 07.

\begin{abstract}
Context: The human Nucleus Accumbens (NA) constitutes nowadays a target of deep brain stimulation (DBS) for selected patients mainly suffering from some psychiatric disorders. Mavridis' Area (MA) of the human brain is the most reliable stereotactically standard part of the NA, regardless of side or gender. Primary purpose of this review article was to discuss the MA as a stereotactic microanatomic target for NA DBS application, emphasizing on technical aspects of targeting the MA.

Evidence Acquisition: A detailed search of the current literature regarding MA was applied. The critically reviewed literature was then used to discuss the MA as a stereotactic microanatomic target for NA DBS application, emphasizing on technical aspects of targeting the MA. The suggestion of future research directions was also aimed.

Results: Following the currently used trajectory through the anterior limb of the internal capsule (ALIC) in combination with the principles of the 'port model', a DBS electrode placement within the NA, targeting the MA, is feasible. MA could be the target area of one, only, of the electrode contacts, preferably contact 1 or 2 . It could be also the target area for contact 0 in ALIC DBS and even the target for a combined NA-ALIC DBS. MA is the first and most representative example of the evolution of stereotactic anatomy into stereotactic microanatomy, in order to serve the development of the field of stereotactic microneurosurgery.

Conclusions: MA is nowadays the most accurate stereotactic anatomic guide for targeting the human NA. It is a representative example of how stereotactic microanatomy could serve the development of stereotactic microneurosurgery. Following the currently used trajectory through the ALIC, a DBS electrode placement within the NA, targeting the MA, is feasible, allowing either NA or ALIC stimulation. Simultaneous stimulation of the NA and ALIC is also possible with this application.
\end{abstract}

Keywords: Deep Brain Stimulation, Mavridis' Area, Nucleus Accumbens, Stereotactic Microanatomy, Stereotactic Microneurosurgery

\section{Context}

The human nucleus accumbens (NA), located at the basal forebrain, is a major part of the ventral striatum and belongs to basal ganglia of the brain. Functioning as a limbic-motor interface, it is connected to limbic and extrapyramidal motor systems (1-6). Figure 1 summarizes the main cortical and subcortical brain structures connected to the human NA $(1,7)$.

The NA is involved in some of the commonest neurologic and psychiatric disorders and constitutes a target of stereotactic and functional neurosurgery for selected patients. These patients mainly suffer from refractory major depression, obsessive-compulsive disorder(OCD), Tourette syndrome and some kinds of addiction (such as heroin addiction and alcoholism), as well as, more recently, anorexia nervosa. NA Deep Brain Stimulation (DBS) is nowadays the main neurosurgical intervention used for treating these patients, with encouraging results (1, 2, 4, 5, 8-11).

Mavridis' Area (MA) (or area M) of the human brain is

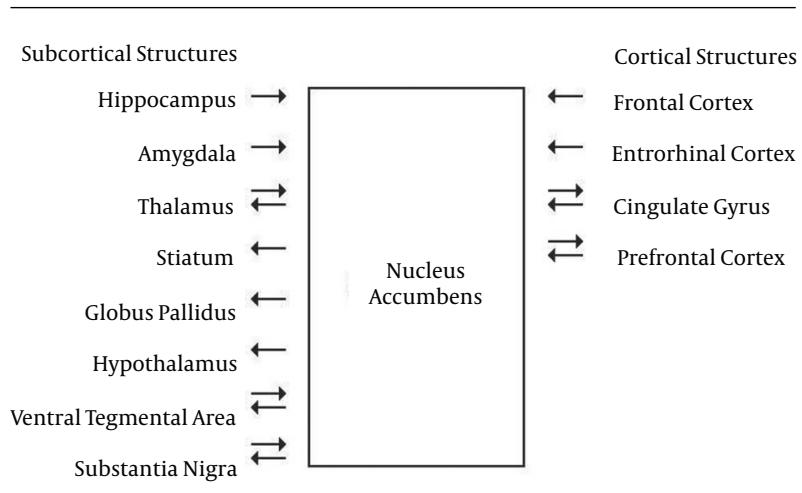

Figure 1. The Main Cortical and Subcortical Connections of the Human Nucleus Accumbens

the stereotactic area defined by coordinates $\left[\mathrm{X}, \mathrm{X}^{\prime}, \mathrm{Y}, \mathrm{Y}^{\prime}, \mathrm{Z}\right.$, $\left.Z^{\prime}\right]=[6,9,2,2,-0.8,-2]$, with the anterior border of the anterior commissure (AC) at the midline defining the stereotactic reference point with coordinates $[\mathrm{X}, \mathrm{Y}, \mathrm{Z}]=[0,0,0]$ 
$(1,12-17)$ (Figure 2$)$. It is the most reliable stereotactically standard part of the human NA, regardless of side or gen$\operatorname{der}(1,13,16)$. MA is the result of mathematical analysis of clinically significant stereotactic coordinates, in a way that mathematical accuracy was translated into microsurgical accuracy (16).

Figure 2. Mavridis' Area (Drawing of Human Brain, Part of Left Hemisphere, Coronal Section at Stereotactic Level $Y=2$ )

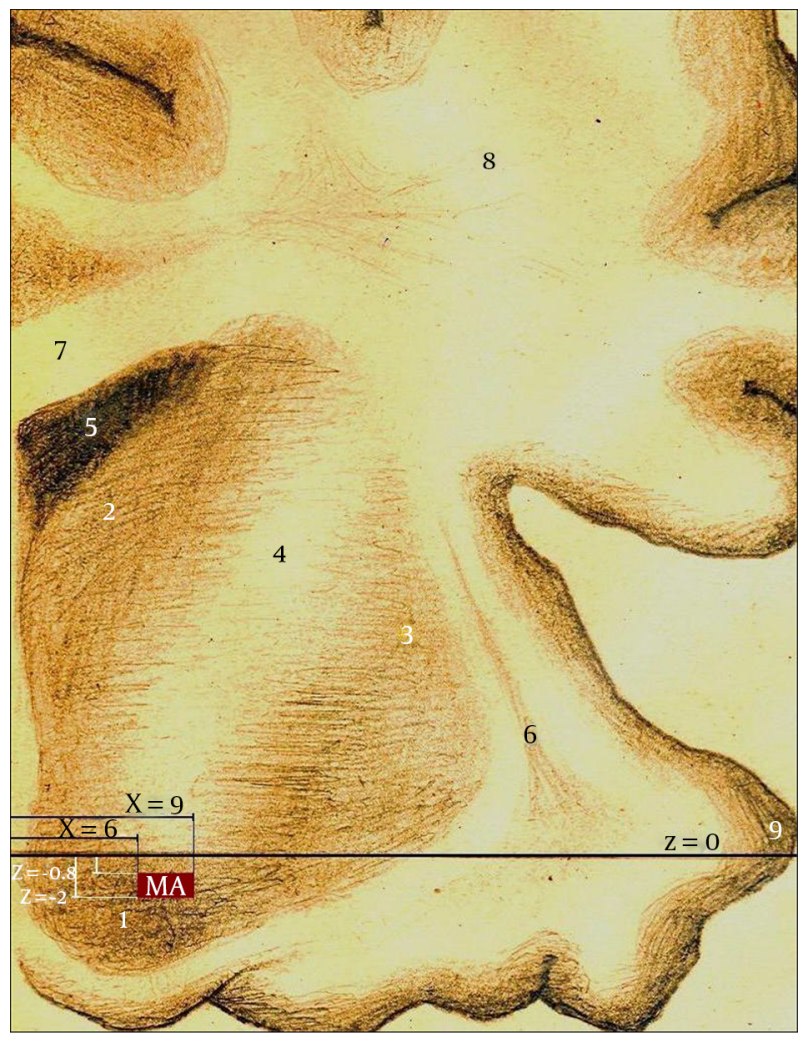

1 , nucleus accumbens; 2 , head of the caudate nucleus; 3 , putamen; 4 , anterior limb of the internal capsule; 5 , frontal horn of the lateral ventricle; 6 , claustrum; 7 , corpus callosum; 8 , frontal lobe (white matter); 9 , insula of Reil (cortex); MA, Mavridis' area; $\mathrm{X}, \mathrm{Y}, \mathrm{Z}$, stereotactic coordinates.

\subsection{Purpose}

Nowadays, MA is the most accurate stereotactic anatomic guide for targeting the human NA (17). It offers a compass for neurosurgeons given the relative variety of NA target coordinates used in the past by several authors (13). Primary purpose of this article was to discuss the MA as a stereotactic microanatomic target for NA DBS application, emphasizing on technical aspects of targeting the MA, as well as to suggest future research directions.

\section{Evidence Acquisition}

A detailed search of the current literature regarding MA was applied, primarily based on articles from the PubMed and Google Scholar databases. Key words for this searching was “Mavridis' area”. Articles regarding human NA DBS applications and reporting target coordinates were also searched, with emphasis on those describing a target area relevant to MA. The critically reviewed literature was then used to discuss the MA as a stereotactic microanatomic target for NA DBS application, emphasizing on technical aspects of targeting the MA. The suggestion of future research directions was also aimed.

\section{Results}

Following the currently used trajectory through the anterior limb of the internal capsule (ALIC) $(1,18)$, in combination with the principles of the 'port model' (safe navigation model) which were recently described in stereotactic anatomic studies $(1,19,20)$, a DBS electrode placement within the NA, targeting the MA, is feasible.

Regarding the electrode models that have been used for human NADBS so far, it should be mentioned that these include Medtronic (Medtronic, Inc., Minneapolis, MN) electrode models 3387, 3389 and 3387 IES $(1,21)$. They all have a diameter of $1.27 \mathrm{~mm}$ and four contacts (poles) $(22,23)$. Models 3387 and 3389 have $1.5 \mathrm{~mm}$ contact length and distance between contacts $1.5 \mathrm{~mm}$ and $0.5 \mathrm{~mm}$, respectively (22). Electrode model 3387 IES has $3 \mathrm{~mm}$ contact length and $4 \mathrm{~mm}$ contact spacing(23). According to these technical details and considering the MA dimensions and its location within the NA (Figure 2), we see that it could be the target area of one, only, of the electrode contacts, preferably contact 1 or 2 ( 0 is the deepest one).

Furthermore, the Medtronic (Medtronic, Inc., Minneapolis, MN) electrode model 3391, used for ALIC DBS in the treatment of chronic, severe and treatment-resistant OCD, has $3 \mathrm{~mm}$ contact length and $4 \mathrm{~mm}$ contact spacing (24). The manufacturer suggests that the trajectory to the target for the deepest lead contact should follow the course of the ALIC toward the point where fibers of the AC cross the ALIC. Interestingly, the suggested range of coordinates for these landmarks is $[\mathrm{X}, \mathrm{Y}, \mathrm{Z}]=[5$ to 10,0 to $5,-5$ to -1$]$, using the AC as stereotactic reference point (24). We see that MA is included in this range area. Consequently, it could be the target area for contact 0 in ALIC DBS.

Additionally, MA could be the electrode target for a combined NA-ALIC DBS, which has been repeatedly described for treating psychiatric disorders. It should be further mentioned that stereotactically standard area $\mathrm{H}$ of the 
human NA has been previously proposed as another (more restricted) target area for this purpose $(1,15)$.

\section{Conclusions}

The new aspect of this article is that it offers a concise review of the current knowledge regarding MA, five years following its identification, as well as a focused analysis of technical aspects of the currently used hardware for NA DBS in relation to MA. The specific methodology which led to the discovery of MA has been previously described (15, 25). Furthermore, comparison of different applied methods for studying NA stereotactic anatomy, as well as their results, has been also previously presented (15).

Due to this point that a precisely in target electrode placement is of paramount importance in DBS of deep brain structures, the use of MA, of course with other factors such as a careful preoperative trajectory planning together will help in minimizing the chances of missing the target.

MA is the first and most representative example of the evolution of stereotactic anatomy into stereotactic microanatomy, in order to serve the modern necessities of stereotactic and functional neurosurgery and specifically the development of the field of Stereotactic Microneurosurgery (17). Future clinical applications of NA DBS, an increasingly used minimally invasive operation, are expected to improve our knowledge of the microenvironment of the NA, as well as the way we approach its targeting and determine stimulation parameters.

A future evolution of electrodes' characteristics could offer smaller lead implants enabling neurosurgeons for even more selective stimulation of specific brain areas, such as nuclear parts instead of whole nuclei. Smaller leads could also contribute in further minimization of specific implantation-related complications such as intracerebral hematomas.

Nowadays, five years after its discovery, MA is the most accurate stereotactic anatomic guide for targeting the human NA. It is the first and most representative example of the evolution of stereotactic anatomy into stereotactic microanatomy, in order to serve the development of Stereotactic Microneurosurgery. Following the currently used trajectory through the ALIC, a DBS electrode placement within the NA, targeting the MA, is feasible, allowing for either NA or ALIC stimulation. For NA DBS, either contact 1 or 2 should be located at the MA, whereas for ALIC DBS, contact 0 has to be located at the MA. Simultaneous stimulation of the NA and ALIC is also possible with this application.

\section{References}

1. Mavridis IN. Stereotactic neurosurgical anatomy of the nucleus accumbens [in Greek]. Athens: National and Kapodistrian University of Athens; 2012.
2. Mavridis I, Anagnostopoulou S. Nucleus accumbens location: anatomical, functional and clinical data [in Greek]. Nevrologia. 2008;17(6):338-46.

3. Mavridis I, Boviatsis E, Anagnostopoulou S. Anatomy of the human nucleus accumbens: a combined morphometric study. Surg Radiol Anat. 2011;33(5):405-14. doi: 10.1007/s00276-010-0766-6. [PubMed: 21203764].

4. Mavridis I, Boviatsis E, Anagnostopoulou S. The human nucleus accumbens suffers parkinsonism-related shrinkage: a novel finding. Surg Radiol Anat. 2011;33(7):595-9. doi: 10.1007/s00276-011-0802-1. [PubMed: 21404044].

5. Mavridis I. The role of the nucleus accumbens in neurological disorders [in Greek]. Neurologia-gr. 2012;21(6):6-11.

6. Mavridis IN. Music and the nucleus accumbens. Surg Radiol Anat. 2015;37(2):121-5. doi: 10.1007/s00276-014-1360-0. [PubMed: 25102783].

7. Mavridis IN, Anagnostopoulou S. Cortical connections of the human nucleus accumbens: measurements and correlations. OA Anat. 2013;1(1):7.

8. Kuhn J, Bührle CP, Lenartz D, Sturm V. Deep brain stimulation in addiction due to psychoactive substance use. Handb Clin Neurol. 2013;116:259-69. doi: 10.1016/B978-0-444-53497-2.00021-8. [PubMed: 24112900].

9. Voges J, Müller U, Bogerts B, Münte T, Heinze HJ. Deep brain stimulation surgery for alcohol addiction. World Neurosurg. 2013;80(3-4):S28. e21-31. doi: 10.1016/j.wneu.2012.07.011. [PubMed: 22824557].

10. Zhang HW, Li DY, Zhao J, Guan YH, Sun BM, Zuo CT. Metabolic imaging of deep brain stimulation in anorexia nervosa: a 18F-FDG PET/CT study. Clin Nucl Med. 2013;38(12):943-8. doi: 10.1097/RLU.0000000000000261. [PubMed: 24212440].

11. Mavridis I, Boviatsis E, Anagnostopoulou S, Sakas DE. The human nucleus accumbens as a target for stereotactic neurosurgery: a combined morphometric study. Acta Neurochir(Wien). 2011;153(3):731.

12. Mavridis IN. Stereotactic neurosurgical anatomy of the nucleus accumbens: four-year outcomes. Surg Radiol Anat. 2013;35(7):637-8. doi: 10.1007/s00276-013-1121-5. [PubMed: 23609422].

13. Mavridis IN. Nucleus accumbens stereotactic surgery: Achieving accuracy through area M. World J Neurol. 2013;3(2):7-9.

14. Mavridis IN. Anatomizing the "King of Neurosciences". World J Neurol. 2013;3(2):4-6.

15. Mavridis I, Boviatsis E, Anagnostopoulou S. Stereotactic anatomy of the human nucleus accumbens: from applied mathematics to microsurgical accuracy. Surg Radiol Anat. 2011;33(7):583-94. doi: 10.1007/s00276-011-0804-z. [PubMed: 21437651].

16. Mavridis IN. Mavridis' area of the human brain: mathematically analysed Anatomy assists Stereotactic Neurosurgery. OA Anat. 2014;2(2):11.

17. Mavridis IN. Anatomic guidance for stereotactic microneurosurgery: a modern necessity and the example of Mavridis' area. Surg Radiol Anat. 2015;37(1):119-20. doi: 10.1007/s00276-014-1323-5. [PubMed: 24957709].

18. Mavridis IN. Approaches and landmarks for nucleus accumbens area: review of the literature and review-based suggestions for nucleus accumbens surgery. OA Anat. 2013;1(1):1.

19. Mavridis I, Anagnostopoulou S. The safest electrode trajectory for deep brain stimulation of the human nucleus accumbens: a stereotactic anatomic study. Minim Invasive Neurosurg. 2011;54(1):16-20. doi: 10.1055/s-0030-1270516. [PubMed: 21506063].

20. Mavridis I. Deep Brain Stimulation of the Nucleus Accumbens: The Safest Electrode Trajectory. 63rd Southern Neurosurgical Society Annual Meeting-Neurosurgery: Next Generation Advice. 2012; Amelia Island, Florida-Program Book. Southern Neurosurgical Society and American Association of Neurological Surgeons; pp. 41-2.

21. Mavridis IN. Nucleus Accumbens stereotaxy for deep brain stimulation: anatomical focus. Acta Neurochir(Wien). 2014;156(3):589-91. doi: 10.1007/s00701-013-1883-x. [PubMed: 24085315]. 
22. Kuhn J, Lenartz D, Huff W, Lee S, Koulousakis A, Klosterkoetter J, et al. Remission of alcohol dependency following deep brain stimulation of the nucleus accumbens: valuable therapeutic implications?. J Neurol Neurosurg Psychiatry. 2007;78(10):1152-3. doi: 10.1136/jnnp.2006.113092. [PubMed: 17878197].

23. Mantione M, van de Brink W, Schuurman PR, Denys D. Smoking cessation and weight loss after chronic deep brain stimulation of the nucleus accumbens: therapeutic and research implications: case report. Neurosurgery. 2010;66(1):E218. doi: 10.1227/01.NEU.0000360570.40339.64. [PubMed: 20023526] dis- cussion E218.

24. Implant manual . Medtronic Reclaim DBS therapy for OCD 3391 Lead kit for deep brain stimulation 2011. [cited 5 October 2014]. Available at:http://www.medtronic.com.hk/wcm/ groups/mdtcom_sg/@mdt/@neuro/documents/documents/http: //www.medtronic.com.hk/wcm/groups/mdtcom_sg.

25. Mavridis I, Boviatsis E, Anagnostopoulou S. Stereotactic anatomy of the nucleus accumbens: analyzing coordinates. 14th European Congress of Neurosurgery. 2011; Rome-Abstracts CD. The European Association of Neurosurgical Societies. 